\title{
Small bowel obstruction following computed tomography and magnetic resonance enterography using psyllium seed husk as an oral contrast agent
}

\author{
Yingming Amy Chen $\mathrm{MD}^{1}$, Patrick Cervini $\mathrm{MD}^{2}$, Anish Kirpalani $M D^{1}$, Paraskevi A Vlachou $M D^{1}$, \\ Samir C Grover MD MEd ${ }^{3}$, Errol Colak MD ${ }^{1}$
}

YA Chen, P Cervini, A Kirpalani, PA Vlachou, SC Grover, E Colak. Small bowel obstruction following computed tomography and magnetic resonance enterography using psyllium seed husk as an oral contrast agent. Can J Gastroenterol Hepatol 2014;28(7):391-395.

The authors report a case series describing four patients who developed small bowel obstruction following the use of psyllium seed husk as an oral contrast agent for computed tomography or magnetic resonance enterography. Radiologists who oversee computed tomography and magnetic resonance enterography should be aware of this potential complication when using psyllium seed husk and other bulking agents, particularly when imaging patients with known or suspected small bowel strictures or active inflammation.

Key Words: CT enterography; MR enterography; Oral contrast

Computed tomography (CT) and magnetic resonance enterography (MRE) play an increasingly critical role in the diagnosis, assessment and management of patients with small bowel disease. CT and MRE have largely replaced fluoroscopic examinations, such as small bowel follow-through and enteroclysis, by providing accurate characterization of the bowel wall and visualization of disease beyond the bowel wall (1-3). Furthermore, CT enterography (CTE) and MRE provide a more comfortable patient experience by circumventing the need for nasojejunal intubation (4).

The diagnostic accuracy of CTE and MRE is predicated on adequate distension of the small bowel by oral contrast. Multiple oral contrast options are available, including psyllium seed husk, which is a nondigestible bulk fibre laxative. To date, there is no established consensus on the ideal oral contrast agent for CTE and MRE.

We report three cases of small bowel obstruction following CTE at Windsor Regional Hospital (Ouellette Campus, Windsor, Ontario) and one case following MRE at St Michael's Hospital (Toronto, Ontario) with psyllium seed husk (Metamucil, Proctor \& Gamble, USA) as the oral contrast agent.

\section{CTE protocol}

\section{EXAMINATION PROTOCOL}

Patients were instructed to be nil per os from midnight and to arrive $1 \mathrm{~h}$ before the examination. Three doses of oral contrast were prepared by diluting $15 \mathrm{~mL}$ Metamucil in $450 \mathrm{~mL}$ of water. Patients were instructed to drink $450 \mathrm{~mL}$ of oral contrast $60 \mathrm{~min}, 40 \mathrm{~min}$ and $20 \mathrm{~min}$ before the examination.

\author{
L'obstruction du grêle après une tomodensitométrie \\ et une entérographie par résonance magnétique \\ faisant appel à l'enveloppe de psyllium comme \\ agent oral de contraste
}

\begin{abstract}
Les auteurs rendent compte d'une série de cas de quatre patients qui ont présenté une obstruction du grêle après avoir consommé l'enveloppe de psyllium comme agent oral de contraste en vue d'une tomodensitométrie ou d'une entérographie par résonance magnétique. Les radiologues qui supervisent les tomodensitométries et les entérographies par résonance magnétique devraient connaître cette complication potentielle lorsqu'ils utilisent l'enveloppe de psyllium et les autres agents gonflants, notamment lorsque les patients qui se soumettent à l'imagerie présentent un rétrécissement connu ou présumé ou une inflammation active du grêle.
\end{abstract}

\begin{abstract}
MRE protocol
Patients were instructed to be on a clear liquid diet after 17:00 the day before the examination, to take an enema (Fleet, CB Fleet, USA) the morning of the examination, and to arrive $2 \mathrm{~h}$ before the examination. Patients were administered $10 \mathrm{mg}$ metoclopramide orally before drinking the Metamucil solution to promote peristalsis. Metamucil at a dose of $1.6 \mathrm{~g} / \mathrm{kg}$ was diluted in $2000 \mathrm{~mL}$ of water. Patients were instructed to drink $500 \mathrm{~mL}$ every $30 \mathrm{~min}$. An additional $10 \mathrm{mg}$ of metoclopramide was administered after drinking $1000 \mathrm{~mL}$ of oral contrast to ensure adequate transit of oral contrast into the small bowel.
\end{abstract}

\section{Case 1}

A 55-year-old woman underwent CTE for the assessment of Crohn disease. CT demonstrated active disease involving $20 \mathrm{~cm}$ of the distal ileum characterized by bowel wall thickening, mucosal hyperenhancement, mural stratification, significant luminal narrowing and mesenteric inflammation (Figure 1A). Approximately $6 \mathrm{~h}$ after CTE, she presented to the emergency department with diffuse abdominal pain. Abdominal radiographs performed $9 \mathrm{~h}$ after CTE demonstrated dilated small bowel with air-fluid levels (Figure 1B). The patient was admitted overnight for conservative treatment and discharged the next day following resolution of symptoms.

Case 2

An 88-year-old man underwent CTE to rule out a small bowel cause of anemia. No small bowel abnormalities were demonstrated by CTE. The patient developed progressively worsening abdominal pain in the

\footnotetext{
${ }^{1}$ Department of Medical Imaging, St Michael's Hospital, Toronto; ${ }^{2}$ Department of Diagnostic Imaging, Windsor Regional Hospital - Ouellette Campus, Windsor; ${ }^{3}$ Division of Gastroenterology, St Michael's Hospital, Toronto, Ontario

Correspondence: Dr Errol Colak, Department of Medical Imaging, St Michael's Hospital, 30 Bond Street, Toronto, Ontario M5B 1 W8.

Telephone 416-864-6060, fax 416-864-5380, e-mail colake@smh.ca

Received for publication March 8, 2014. Accepted April 3, 2014
} 


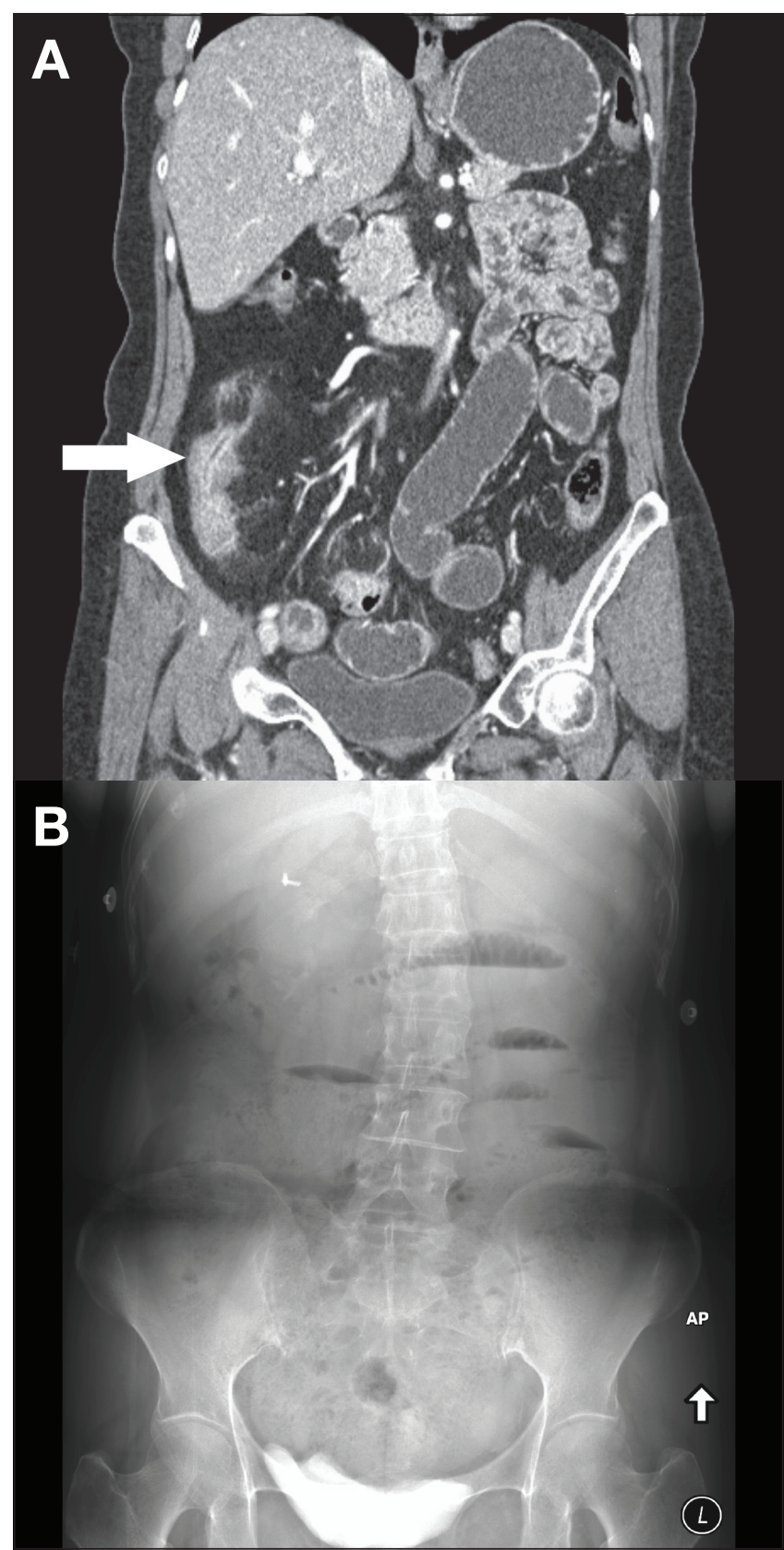

Figure 1) A Coronal computed tomography enterography image demonstrating active inflammation of the terminal ileum (arrow). The upstream small bowel is filled with oral contrast. B Upright abdominal radiograph performed $9 \mathrm{~h}$ later demonstrating dilated small bowel with air-fluid levels consist with a small bowel obstruction

hours that followed the examination and returned to hospital the next morning. Abdominal radiographs were consistent with a small bowel obstruction (Figure 2). The patient improved with conservative management and his symptoms resolved by the next day.

\section{Case 3}

A 61-year-old woman with a presumed history of ulcerative colitis and remote total colectomy underwent CTE for symptoms of intermittent bowel obstruction. The patient was treated conservatively for a small bowel obstruction three weeks earlier. CTE revealed a right lower quadrant parastomal hernia with active inflammation of the immediately upstream small bowel (Figures $3 \mathrm{~A}$ and $3 \mathrm{~B}$ ). The remainder of the small bowel was unremarkable. The patient presented $10 \mathrm{~h}$ later with
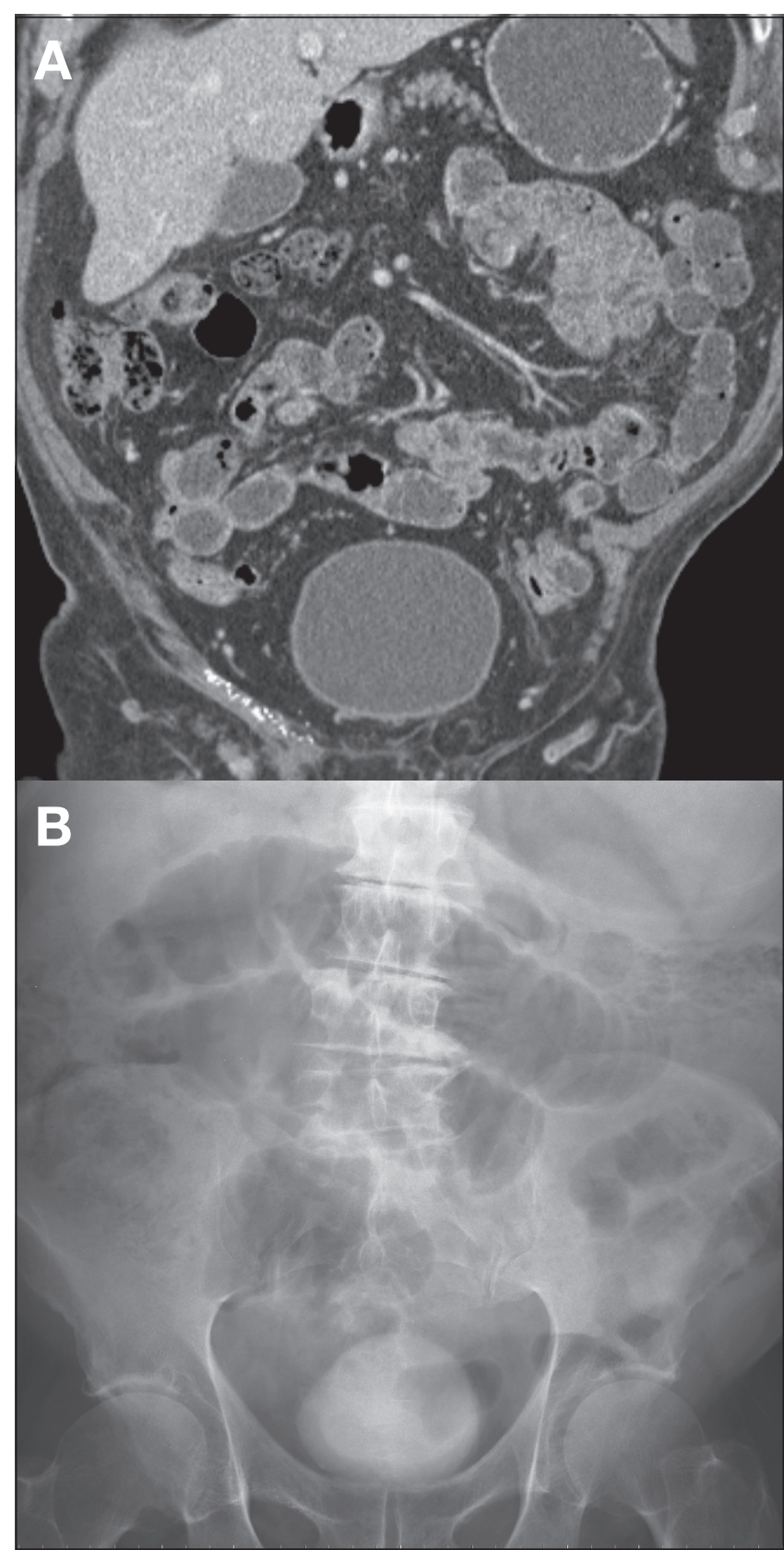

Figure 2) A Coronal image from a normal computed tomography enterogram. B Supine abdominal radiograph performed the subsequent day demonstrating multiple distended loops of small bowel

generalized abdominal pain and nausea. Repeat CT scan of the abdomen showed diffuse small bowel dilation with a transition point at the parastomal hernia (Figures 3C and 3D). The patient did not improve with conservative management and exploratory laparotomy was undertaken the next day. Multiple adhesions were found around the stoma that resulted in kinking of the adjacent bowel. An ischemic segment of bowel was resected and a new stoma was created. There were no perioperative complications and the patient was discharged in stable condition.

\section{Case 4}

A 51-year-old man with a history of Crohn disease and distal ileal resection underwent MRE that demonstrated a long segment of narrowing of the efferent limb of the side-to-side ileoileal anastomosis 


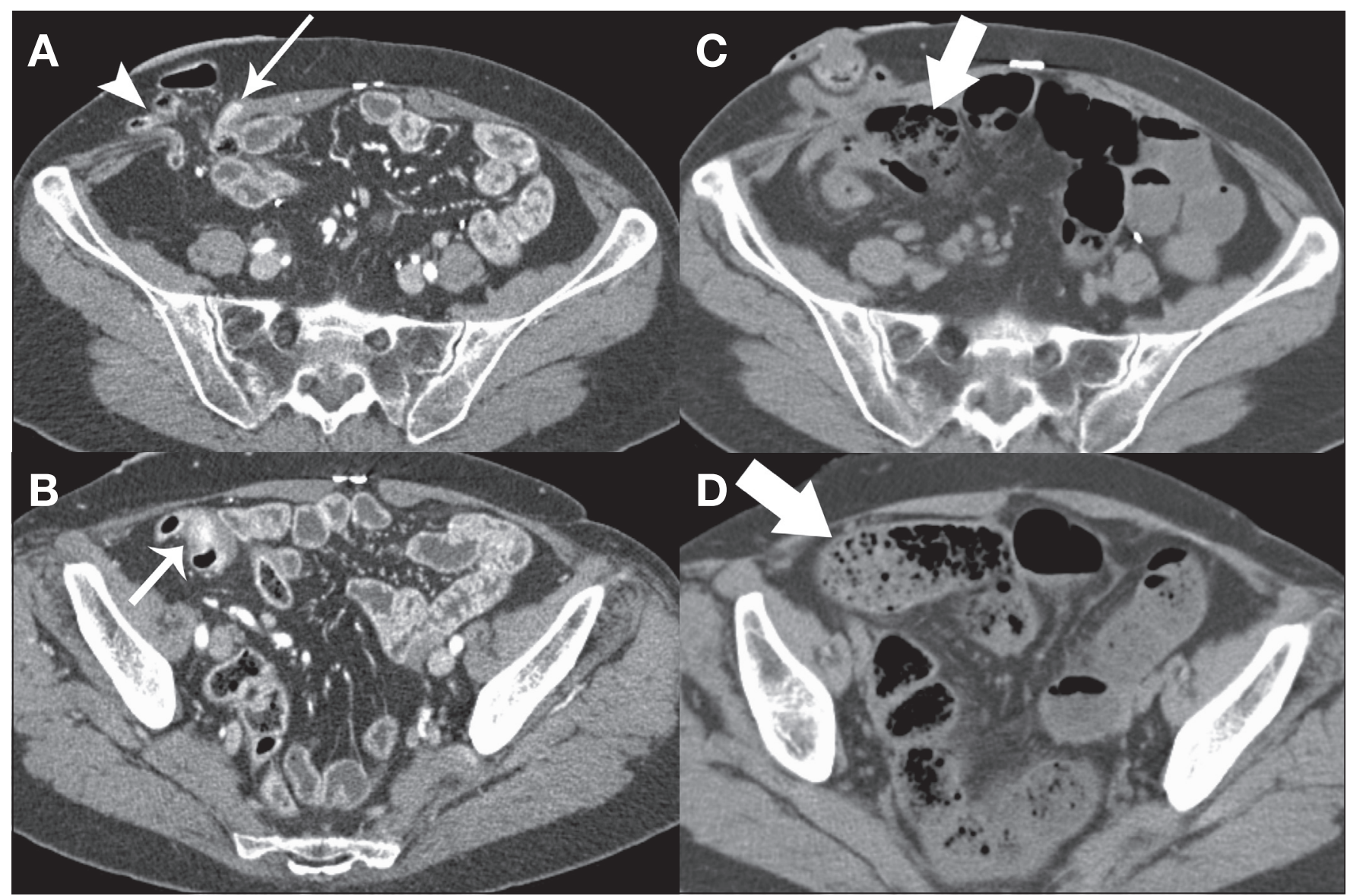

Figure 3) A and B Axial computed tomography enterography images demonstrating a right lower quadrant parastomal hernia (arrowhead) with active inflammation of the immediately upstream small bowel (thin arrows). C and D Computed tomography scan performed $10 \mathrm{~h}$ later demonstrates diffuse small bowel dilation and fecalization of small bowel content (thick arrows) to the level of a transition point in the parastomal hernia

(Figure 4A). There were no imaging features to suggest active small bowel inflammation. Approximately $12 \mathrm{~h}$ after the examination, the patient developed severe nausea and vomiting, diffuse abdominal pain and bloating. A CT scan of the abdomen demonstrated a high-grade small bowel obstruction with a transition point just beyond the site of the ileoileal anastomosis at the narrowed segment of the efferent limb (Figure 4B). He was managed conservatively by nasogastric intubation and bowel rest. His symptoms resolved completely by $48 \mathrm{~h}$.

\section{DISCUSSION}

CTE and MRE have emerged as important investigations for small bowel disorders $(2,3)$, and in influencing clinical management of Crohn disease $(5,6)$. Established indications for CTE and MRE include staging and follow-up of inflammatory bowel disease and its complications, evaluation of obscure gastrointestinal bleeding and investigation of small bowel tumours (7). Emerging uses include evaluation of diffuse small bowel diseases, such as celiac disease (8) and vasculitis, as well as screening of strictures before capsule endoscopy (9). The added advantages of MRE include improved tissue contrast and lack of ionizing radiation, making it ideal for younger patients with inflammatory bowel disease who often require repeated cross-sectional imaging. Furthermore, MRE is capable of real-time functional imaging, which assesses small bowel peristaltic patterns in gastrointestinal motility disorders (10).

Bowel distension is essential for small bowel imaging because collapsed bowel may mimic or obscure small bowel pathology. To date, there is no established consensus on the ideal oral contrast agent for CTE and MRE. Multiple oral contrast agents are available, with the choice varying among institutions based on extent of bowel distension, uniformity of luminal opacification, availability, ease of use, cost and side-effect profile. CTE and MRE oral contrast agents are either hyperosmolar agents that prevent the absorption of water from the small bowel or bulking agents that result in small bowel distension.

The most commonly used oral agents are polyethylene glycol and low-density barium suspensions such as VoLumen (Bracco Diagnostics, USA) with mannitol, sorbitol, methylcellulose, locust bean gum and bulk fibre laxatives representing less commonly used options (11).

While VoLumen is a commonly used oral contrast agent in many jurisdictions, it has not been approved by Health Canada (the Canadian drug regulatory agency) for use as an oral contrast agent. We have anecdotally observed variable small bowel distension and gastrointestinal tract transit time when using polyethylene glycol as an oral contrast agent.

Psyllium seed husk products, including Metamucil, are commonly used bulking laxative and fibre supplement products. When mixed with water, psyllium can function as an oral contrast agent by forming a nondigestible hydrogel that absorbs water and subsequently distends the small bowel (12). It is relatively inexpensive, easy to prepare and administer, and well-tolerated by patients. Reported side effects are typically mild and include bloating, flatulence and diarrhea (13). The reported experience of other centres (12) supported its performance and safety as an oral contrast agent. Consequently, Metamucil was being used as the oral contrast agent for CTE at Windsor Regional Hospital (Ouellette Campus) and MRE at St Michael's Hospital.

Small bowel obstruction following use of psyllium is an uncommon occurrence. Only two cases of small bowel obstruction have been reported after CTE or MRE when using Metamucil or a psyllium-equivalent oral 


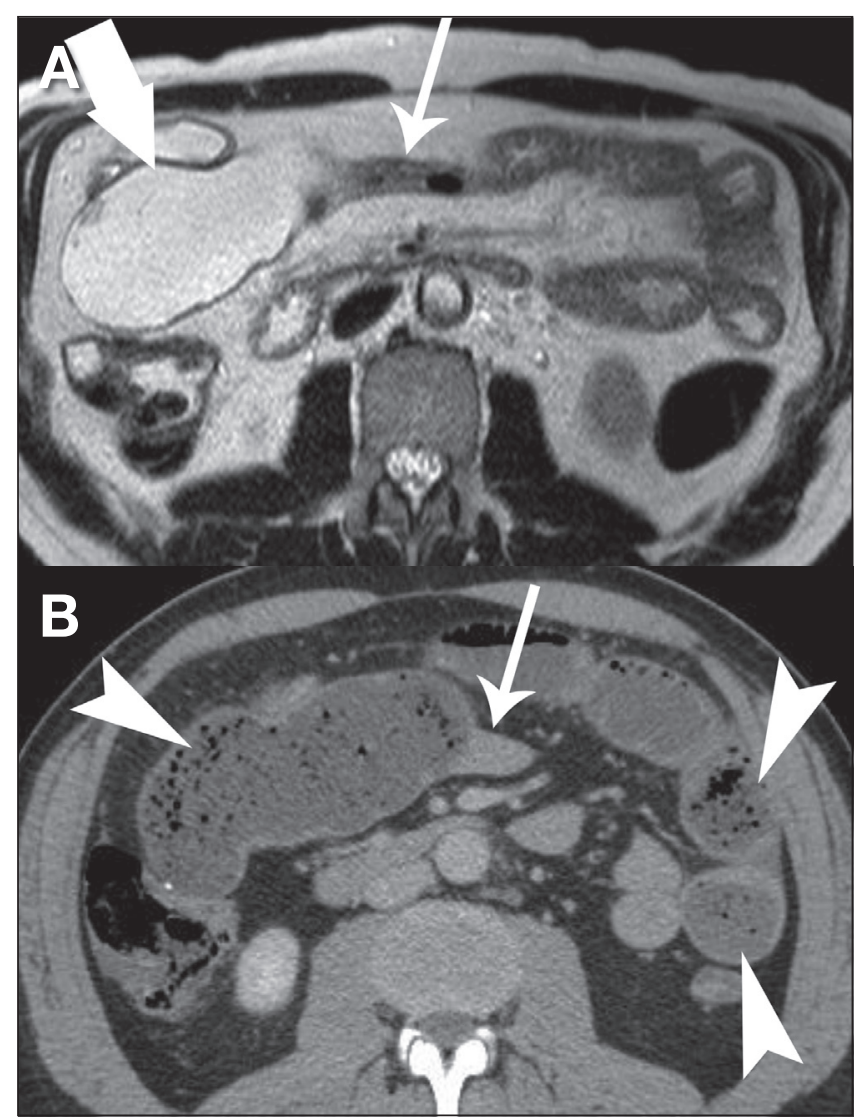

Figure 4) A Axial T2-weighted magnetic resonance enterography image demonstrating a long segment of narrowing (thin arrow) distal to an ileoileal anastomosis (thick arrow) without evidence of active inflammation. B Computed tomography imaging performed $12 \mathrm{~h}$ later showing diffuse dilation of the small bowel with fecalization of bowel content (arrowheads) and a transition point at the narrowed segment of bowel demonstrated on magnetic resonance enterography (thin arrow)

contrast agent $(14,15)$. The time course between psyllium ingestion to the development of small bowel obstruction was $12 \mathrm{~h}$ in one case and 'several days' in the other. Neither circumstances were well described nor had subsequent imaging available to confirm the obstruction. Several other trials evaluating the side effects of psyllium and other bulking agents on healthy individuals did not report any severe complications $(4,11,12,15-18)$.

Psyllium bezoar causing small bowel obstruction has been reported in elderly populations that chronically use high concentrations of psyllium without adequate water consumption $(19,20)$. Incidents of psyllium bezoar causing esophageal obstruction have also been described in patients who ingest dry psyllium preparations without adequate water (21-24).

We suspect that the presence of small bowel strictures represents a risk factor for obstruction following the use of psyllium as an oral contrast agent. The transition point of the small bowel obstruction in one of our patients occurred at an active segment of Crohn disease in which there was significant luminal narrowing. Transition points in two other patients occurred at a narrowed surgical anastomotic site and within a parastomal hernia. The Metamucil-water suspension may have formed a semisolid mucilaginous mass, causing intrinsic obstruction at these points with higher predisposition to obstruction due to narrowing.

As a result of our experience, we suggest that centres exercise caution when using psyllium or other bulking agents as an oral contrast agent in patients with known or suspected small bowel strictures or active inflammation. For centres that continue to use psyllium or other bulking agents, patients should be informed of the potential risk of obstruction and of symptoms that should prompt them to seek medical attention. Metamucil has been replaced by a 3\% sorbitol solution for both CTE and MRE examinations at our institutions, with no negative impact on small bowel distension (25), patient acceptance and side effects, or departmental workflow.

\section{CONCLUSION}

We report a case series describing four patients who developed small bowel obstruction following the use of psyllium seed husk (Metamucil) as an oral contrast agent for CTE or MRE. Radiologists who oversee CTE and MRE examinations should be aware of the potential complication of Metamucil and other bulking agents, particularly when imaging patients with known or suspected small bowel strictures or active inflammation.

DISCLOSURES: The authors have no financial disclosures or conflicts of interest to declare.

\section{REFERENCES}

1. Bernstein CN, Greenberg H, Boult I, Chubey S, Leblanc C, Ryner L. A prospective comparison study of MRI versus small bowel followthrough in recurrent Crohn's disease. Am J Gastroenterol 2005;100:2493-502.

2. Hara AK, Leighton JA, Heigh RI, et al. Crohn disease of the small bowel: Preliminary comparison among CT enterography, capsule endoscopy, small-bowel follow-through, and ileoscopy. Radiology 2006;238:128-34.

3. Lee SS, Kim AY, Yang SK, et al. Crohn disease of the small bowel: Comparison of CT enterography, MR enterography, and smallbowel follow-through as diagnostic techniques. Radiology 2009;251:751-61.

4. Frokjaer JB, Larsen E, Steffensen E, Nielsen AH, Drewes AM. Magnetic resonance imaging of the small bowel in Crohn's disease. Scand J Gastroenterol 2005;40:832-42.

5. Ha CY, Kumar N, Raptis CA, Narra VR, Ciorba MA. Magnetic resonance enterography: Safe and effective imaging for stricturing Crohn's disease. Dig Dis Sci 2011;56:2906-13.

6. Messaris E, Chandolias N, Grand D, Pricolo V. Role of magnetic resonance enterography in the management of Crohn disease. Arch Surg 2010;145:471-5.

7. Masselli G, Gualdi G. CT and MR enterography in evaluating small bowel diseases: When to use which modality? Abdom Imaging 2013;38:249-59.

8. Tennyson CA, Semrad CE. Small bowel imaging in celiac disease. Gastrointest Endosc Clin N Am 2012;22:735-46.

9. Fork FT, Aabakken L. Capsule enteroscopy and radiology of the small intestine. Eur Radiol 2007;17:3103-11.

10. Amzallag-Bellenger E, Oudjit A, Ruiz A, Cadiot G, Soyer PA, Hoeffel CC. Effectiveness of MR enterography for the assessment of small-bowel diseases beyond Crohn disease. Radiographics 2012;32:1423-44.

11. Lauenstein TC, Schneemann H, Vogt FM, Herborn CU, Ruhm SG, Debatin JF. Optimization of oral contrast agents for MR imaging of the small bowel. Radiology 2003;228:279-83.

12. Patak MA, Froehlich JM, von Weymarn C, Ritz MA, Zollikofer CL, Wentz K. Non-invasive distension of the small bowel for magneticresonance imaging. Lancet 2001;358 987-8.

13. Singh B. Psyllium as therapeutic and drug delivery agent. Int J Pharm 2007;334:1-14.

14. Doerfler OC, Ruppert-Kohlmayr AJ, Reittner P, Hinterleitner T, Petritsch W, Szolar DH. Helical CT of the small bowel with an alternative oral contrast material in patients with Crohn disease. Abdom Imaging 2003;28:313-8.

15. Reittner P, Goritschnig T, Petritsch W, et al. Multiplanar spiral CT enterography in patients with Crohn's disease using a negative oral contrast material: Initial results of a noninvasive imaging approach. Eur Radiol 2002;12:2253-7.

16. Ajaj W, Goehde SC, Schneemann H, Ruehm SG, Debatin JF, Lauenstein TC. Oral contrast agents for small bowel MRI: Comparison of different additives to optimize bowel distension. Eur Radiol 2004;14:458-64. 
17. Froehlich JM, Daenzer M, von Weymarn C, Erturk SM, Zollikofer CL, Patak MA. Aperistaltic effect of hyoscine N-butylbromide versus glucagon on the small bowel assessed by magnetic resonance imaging. Eur Radiol 2009;19:1387-93.

18. Jensen MD, Nathan T, Kjeldsen J, Rafaelsen SR. Incidental findings at MRI-enterography in patients with suspected or known Crohn's disease. World J Gastroenterol 2010;16:76-82.

19. Fisher RE. Psyllium seeds: Intestinal Obstruction. Cal West Med 1938;48:190

20. Frohna WJ. Metamucil bezoar: An unusual cause of small bowel obstruction. Am J Emerg Med 1992;10:393-5.

21. Herrle F, Peters T, Lang C, von Fluee M, Kern B, Peterli R. Bolus obstruction of pouch outlet by a granular bulk laxative after gastric banding. Obes Surg 2004;14:1022-4.
22. Perez-Piqueras J, Silva C, Jaqueti J, et al. Endoscopic diagnosis and treatment of an esophageal bezoar resulting from bulk laxative ingestion. Endoscopy 1994;26:710.

23. Sauerbruch T, Kuntzen O, Unger W. Agiolax bolus in the esophagus. Report of two cases. Endoscopy 1980;12:83-5.

24. Veronelli A, Ranieri R, Laneri M, et al. Gastric bezoars after adjustable gastric banding. Obes Surg 2004;14:796-7.

25. Saini S, Colak E, Anthwal S, Vlachou PA, Raikhlin A, Kirpalani A. Comparison of $3 \%$ sorbitol versus psyllium fibre as oral contrast agents in magnetic resonance enterography. Society of Abdominal Radiology 2014 Annual Scientific Meeting and Educational Course, Boca Raton, 2014. 


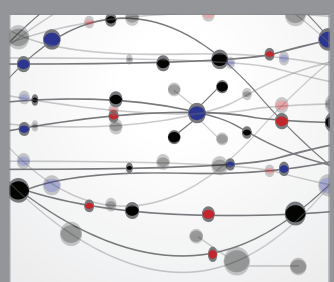

The Scientific World Journal
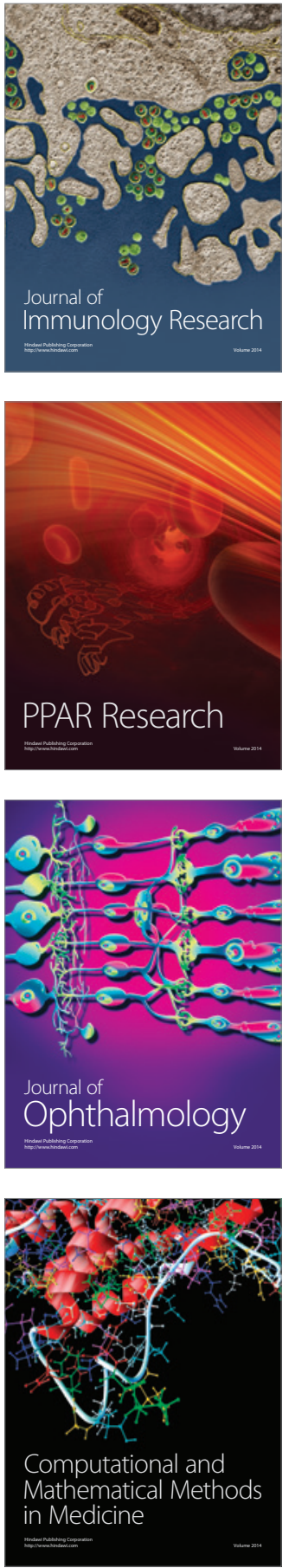

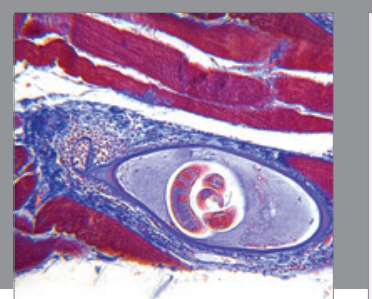

Gastroenterology Research and Practice

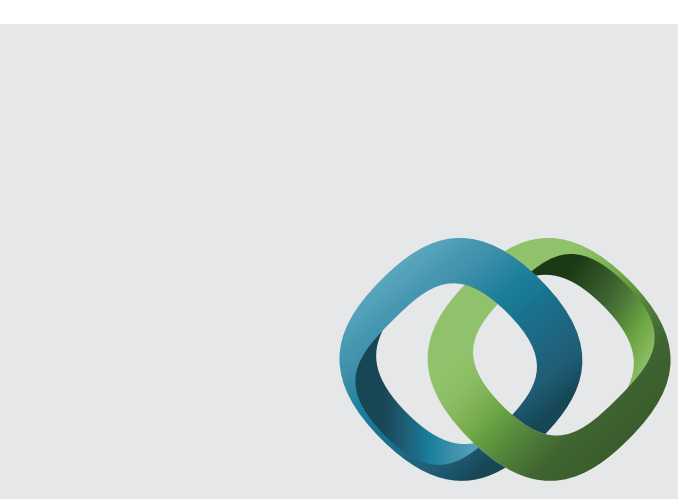

\section{Hindawi}

Submit your manuscripts at

http://www.hindawi.com
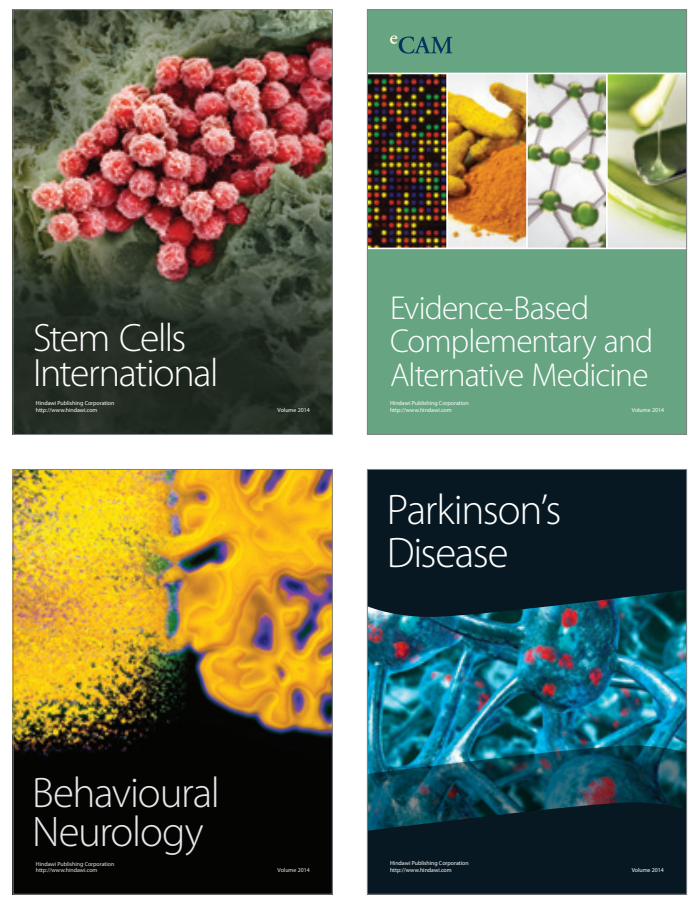
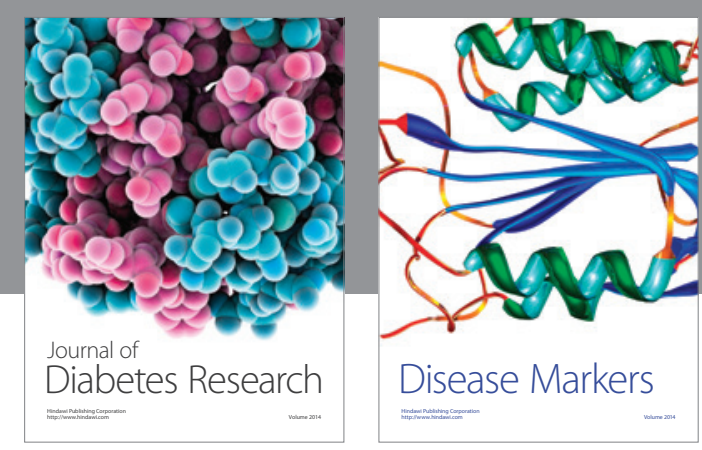

Disease Markers
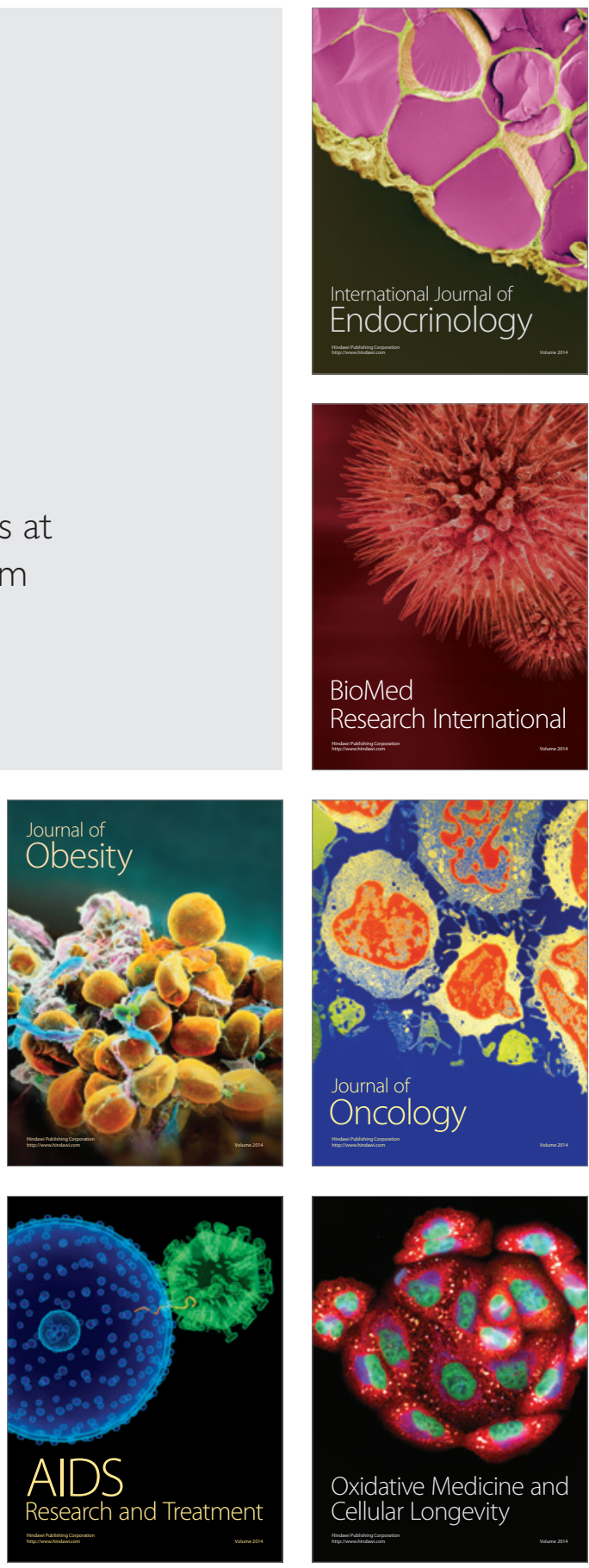